\title{
EFFECTS OF INTRA-AMNIOTIC ENDOTOXIN EXPOSURE ON POSTNATAL KIDNEY DEVELOPMENT IN RATS
}

\author{
H. Takahashi ${ }^{1}$, K. Suzuki ${ }^{2}$, H. Masaki ${ }^{3}$, M. Tamura ${ }^{2}$ \\ ${ }^{l}$ Neonatology, Tokyo Metropolitan Children's Hospital, Fuchu, ${ }^{2}$ Neonatology, Saitama Medical Center, \\ Kawagoe, ${ }^{3}$ Pediatrcs, St. Mariannna University, School of Medicine, Kawasaki, Japan
}

Background and aims: Chorioamnionitis (CAM) is one of the major causes of preterm delivery and also known to be associated with impairment of various developing organs. However, it is not clear whether CAM affects development of the kidney. The objective was to study effects of intra-amniotic lipopolysaccharide (LPS) on development of the kidney.

Methods: At $20 \mathrm{~d}$ gestation, pregnant SD rats were anesthetized and the uterus exposed under general anesthesia. The uterine wall was punctured and $0.1 \mu \mathrm{g}$ LPS; E.coli endotoxin (O55:B5;Sigma,St Louis,MO,USA) dissolved in $0.1 \mathrm{ml}$ saline injected into each amniotic cavity (L group). In the control group (C group), $0.1 \mathrm{ml}$ saline was injected. At $22 \mathrm{~d}$ (term), the fetuses were delivered spontaneously and vaginally. At postnatal day 56, rats were euthanized and the left kidney was collected. The kidney was processed and stained with hematoxylin-eosin for histological examination. We measured the numerical density of the glomeruli in the cortex (glomerular number/cortical volume $(\mathrm{Ng} / \mathrm{C})$ and the mean glomerular volume $(\mathrm{Vg})$ using morphometric method.

Results: There were no significant differences in body weight and kidney weight between $\mathrm{C}$ and $\mathrm{L}$ groups. Only in males, $\mathrm{Ng} / \mathrm{C}$ was lower(C vs L: $84 \pm 17$ vs $33 \pm 4$, mean $\left.\pm \mathrm{SE}, / \mathrm{mm}^{3}, \mathrm{p}<0.05\right)$ and $\mathrm{Vg}$ was higher (C vs $\mathrm{L}: 30 \pm 4$ vs $44 \pm 6$, mean $\left.\pm \mathrm{SE}, \times 10^{-5} \mathrm{~mm}^{3}, \mathrm{p}<0.05\right)$ in the $\mathrm{L}$ group.

Conclusions: Antenatal intra-amniotic LPS may affect nephron endowment, which may be influenced by gender. 